\title{
Acoustically Active Antibubbles
}

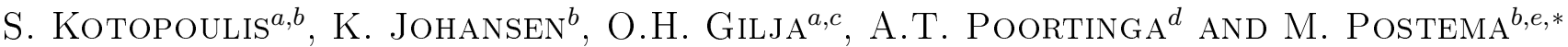 ${ }^{a}$ National Centre for Ultrasound in Gastroenterology, Haukeland University Hospital, Jonas Lies vei 65,5021 Bergen, Norway}

${ }^{b}$ Department of Physics and Technology, University of Bergen, Allégaten 55, 5007 Bergen, Norway

${ }^{c}$ Department of Clinical Medicine, University of Bergen, Jonas Lies vei 65, 5021 Bergen, Norway

${ }^{d}$ Department of Mechanical Engineering, Eindhoven University of Technology, 5612 AZ Eindhoven, The Netherlands ${ }^{e}$ The Michelsen Centre for Industrial Measurement Science and Technology, Fantoftvegen 38, 5892 Bergen, Norway

\begin{abstract}
In this study, we analyse the behaviour of antibubbles when subjected to an ultrasonic pulse. Specifically, we derive oscillating behaviour of acoustic antibubbles with a negligible outer shell, resulting in a Rayleigh-Plesset equation of antibubble dynamics. Furthermore, we compare theoretical behaviour of antibubbles to behaviour of regular gas bubbles. We conclude that antibubbles and regular bubbles respond to an acoustic wave in a very similar manner if the antibubble's liquid core radius is less than half the antibubble radius. For larger cores, antibubbles demonstrate highly harmonic behaviour, which would make them suitable vehicles in ultrasonic imaging and ultrasound-guided drug delivery.
\end{abstract}

DOI: $10.12693 /$ APhysPolA.127.99

PACS: 47.55.dd, 43.25.Yw, 43.80.Sh

\section{Introduction}

Ultrasound contrast agents consist of microscopically small gas bubbles, encapsulated by elastic shells. These agents are commonly used in clinical diagnostic imaging [1].

Sonoporation, the transient formation of micron-sized pores in cell membranes induced by ultrasound in combination with microbubbles is of great interest for targeted drug and gene delivery [2]. This technique shows promise in cancer treatment as it allows increased localised drug delivery $[3,4]$. Nevertheless, during sonoporation treatment, patients are still affected by systemic side effects as the chemotherapeutics are still systemically delivered. An ideal solution to this problem would be to encapsulate a therapeutic load into a delivery tool and to release it at the desired location.

Many attempts have been undertaken to incorporate chemotherapeutics into microbubbles [5]. The most common technique is to embed or bond a therapeutic load into the microbubbles' shell [6, 7]. However, adding a viscoelastic layer to a microbubble greatly impedes its oscillation amplitude, making it challenging to disrupt and release its therapeutic load at low acoustic amplitudes. A solution would be to incorporate the therapeutic load into the gas core of the microbubble. Such antibubbles should allow for easy manipulation and disruption in sound fields [8].

Stable, micron-sized antibubbles have already been produced. These have a load volume of approximately

* corresponding author; e-mail: michiel.postema@uib.no half the entire bubble volume, significantly higher than with any other loading method [9, 10]. Figure 1 shows an optical microscopy image of stable antibubbles in solution.

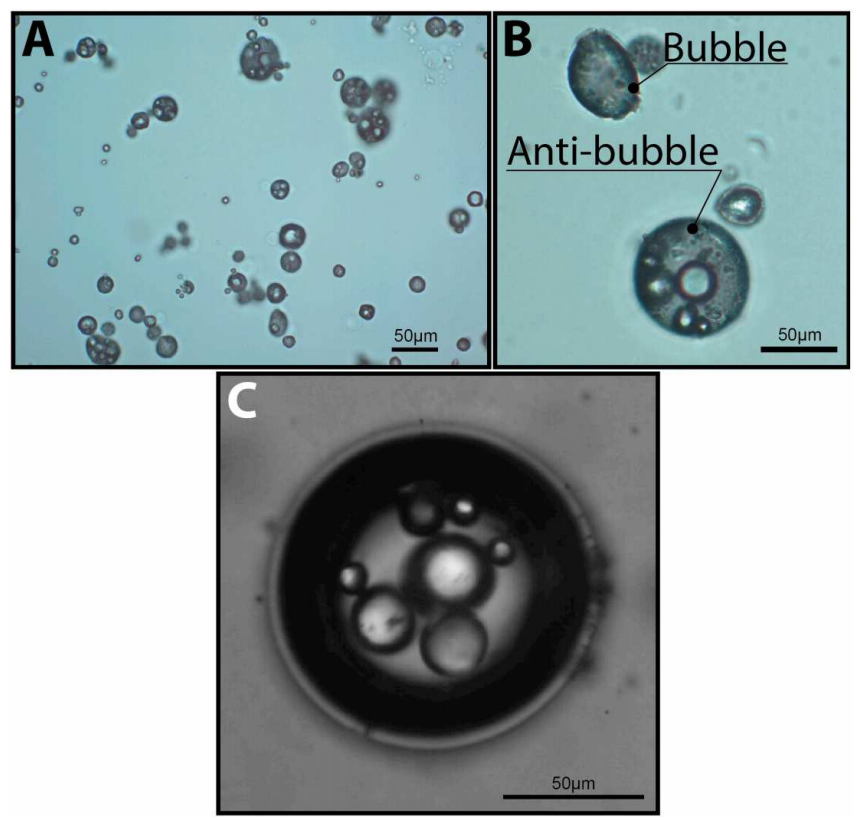

Fig. 1. Optical microscopy image of stable antibubbles with single and multiple cores.

In this study, we examine oscillating behaviour of acoustic antibubbles resulting in a Rayleigh-Plesset-like equation of bubble dynamics. Furthermore, we compare the theoretical behaviour of antibubbles to that of regular gas bubbles. 


\section{Theory}

\subsection{Fundamental equation of antibubble dynamics}

Let us consider a polytropic gas encapsulation with a negligible outer shell and a spherical incompressible liquid core. The following unstable equilibrium can be formulated:

$$
p_{\mathrm{g} 0}+p_{\mathrm{v}}=p_{0}+2 \sigma / R_{0},
$$

where $p_{\mathrm{g} 0}$ is the initial gas pressure, $p_{\mathrm{v}}$ is the vapour pressure, $p_{0}$ is the ambient pressure, $\sigma$ is the surface tension, and $R_{0}$ is the initial outer radius of the microbubble. Assuming a pressure change in the surrounding fluid under adiabatic conditions inside the bubble,

$$
p_{\mathrm{g} 0} V_{0}^{\gamma}=p_{\mathrm{g}} V^{\gamma}
$$

where $V_{0}$ is the initial gas volume, $\gamma$ is the polytropic exponent of the gas, $p_{\mathrm{g}}$ is the instantaneous gas pressure, and $V$ is the instantaneous gas volume. By introducing the instantaneous absolute pressure at the bubble wall $p_{\mathrm{L}}$, the droplet radius $R_{\mathrm{d}}$, and the instantaneous bubble radius $R$, Eqs. (2.1.1) and (2.1.2) can be combined to

$$
p_{\mathrm{L}}=\left(p_{0}-p_{\mathrm{v}}+\frac{2 \sigma}{R_{0}}\right)\left(\frac{R_{0}^{3}-R_{\mathrm{d}}^{3}}{R^{3}-R_{\mathrm{d}}^{3}}\right)^{\gamma}+p_{\mathrm{v}}-\frac{2 \sigma}{R} .
$$

If the instantaneous pressure in the surrounding fluid is created from a sound wave, the bubble will respond with volumetric oscillations. During time $\Delta t$, the liquid mass flowing outside the bubble across a surface with radius $r$ must be equal to the mass displaced by the expanding or contracting antibubble

$$
4 \pi r^{2} \rho(\partial r / \partial t) \Delta t=4 \pi R^{2} \rho(\partial R / \partial t) \Delta t,
$$

where $\rho$ is the density of the liquid surrounding the bubble, and $\partial r / \partial t$ is the particle velocity. The work done by the oscillating antibubble must equal the kinetic energy of the surrounding fluid

$$
\begin{gathered}
4 \pi \int_{R_{0}}^{R}\left(p_{\mathrm{L}}-p_{0}\right) R^{2} \mathrm{~d} R= \\
2 \pi \rho \int_{R}^{\infty}(\partial r / \partial t)^{2} r^{2} \mathrm{~d} t .
\end{gathered}
$$

Rearranging the right-hand side of (2.1.5) by substituting (2.1.4) for the particle velocity, the kinetic energy of the surrounding fluid, $E_{\mathrm{k}}$, becomes

$$
E_{\mathrm{k}}=2 \pi \rho R^{3}(\partial R / \partial t)^{2} .
$$

After substitution of

$$
\frac{\partial}{\partial R}\left(\frac{\partial R}{\partial t}\right)^{2}=2 \frac{\partial^{2} R}{\partial t^{2}}
$$

into (2.1.6), (2.1.5) becomes the fundamental equation of bubble dynamics [11]:

$$
\frac{p_{\mathrm{L}}-p_{0}}{\rho}=R \frac{\partial^{2} R}{\partial t^{2}}+\frac{3}{2}\left(\frac{\partial R}{\partial t}\right)^{2} .
$$

After introducing a driving function $P(t)$ and replacing $p_{\mathrm{L}}$ by (2.1.3), the fundamental equation of antibubble dynamics becomes

$$
R \frac{\partial^{2} R}{\partial t^{2}}+\frac{3}{2}\left(\frac{\partial R}{\partial t}\right)^{2}=\frac{1}{\rho}\left[\left(p_{0}-p_{\mathrm{v}}+\frac{2 \sigma}{R_{0}}\right)\right.
$$

$$
\left.\times\left(\frac{R_{0}^{3}-R_{\mathrm{d}}^{3}}{R^{3}-R_{\mathrm{d}}^{3}}\right)^{\gamma}+p_{\mathrm{v}}-\frac{2 \sigma}{R}-p_{0}-P(t)\right] .
$$

\subsection{Dynamics of antibubbles in a Newtonian viscous fluid}

The viscosity $\eta$ of a Newtonian viscous fluid equals by definition the rate of strain $\Delta \varepsilon / \Delta t$. In stress analysis, the principal stresses are defined positive for expanding media, as opposed to in fluid physics. For an instantaneous hydrostatic pressure $p$, for an incompressible liquid,

$$
p_{\mathrm{L}}=-p-2 \eta\left(\partial \varepsilon_{\mathrm{r}} / \partial t\right),
$$

where $\varepsilon_{\mathrm{r}}$ is the radial strain [10]. Using (2.1.4), the radial rate of strain can be expressed in terms of $r$ and $R$ :

$$
\frac{\partial \varepsilon_{\mathrm{r}}}{\partial t}=-\frac{2 R^{2}}{r^{3}} \frac{\partial R}{\partial t}
$$

At the antibubble surface $r=R,(2.2 .2)$ simplifies to

$$
\frac{\partial \varepsilon_{\mathrm{r}}}{\partial t}=-\frac{2}{R} \frac{\partial R}{\partial t}
$$

Combining (2.1.8), (2.2.1), and (2.2.2) results in the fundamental equation of the dynamics of bubbles in a Newtonian viscous fluid

$$
\frac{1}{\rho}\left(p_{\mathrm{L}}-p_{0}-\frac{4 \eta}{R} \frac{\partial R}{\partial t}\right)=R \frac{\partial^{2} R}{\partial t^{2}}+\frac{3}{2}\left(\frac{\partial R}{\partial t}\right)^{2}
$$

After introducing a driving function $P(t)$ and replacing $p_{\mathrm{L}}$ by (2.1.3), the fundamental equation of antibubble dynamics becomes

$$
\begin{aligned}
& R \frac{\partial^{2} R}{\partial t^{2}}+\frac{3}{2}\left(\frac{\partial R}{\partial t}\right)^{2}=\frac{1}{\rho}\left[\left(p_{0}-p_{\mathrm{v}}+\frac{2 \sigma}{R_{0}}\right)\right. \\
& \left.\quad \times\left(\frac{R_{0}^{3}-R_{\mathrm{d}}^{3}}{R^{3}-R_{\mathrm{d}}^{3}}\right)^{\gamma}+p_{\mathrm{v}}-\frac{2 \sigma}{R}-\frac{4 \eta}{R} \frac{\partial R}{\partial t}-p_{0}-P(t)\right] .
\end{aligned}
$$

This is the Rayleigh-Plesset equation for antibubbles in a Newtonian viscous fluid, which can only be applied if the surrounding medium is incompressible and the gas is polytropic.

If we assume (2.2.4) to have a solution of the form

$$
R(t)=R_{0}(1+\chi(t)),
$$

where $\chi(t) \ll R_{0}$, we can linearize (2.2.4) and find the damped resonance frequency for an antibubble

$$
\begin{aligned}
\omega_{\mathrm{d}}^{2} & =\frac{1}{R_{0}^{2} \rho}\left\{3 \gamma\left(p_{0}-p_{\mathrm{v}}+\frac{2 \sigma}{R_{0}}\right) /\left[1-\left(\frac{R_{\mathrm{d}}}{R_{0}}\right)^{3}\right]\right. \\
& \left.-\frac{2 \sigma}{R_{0}}-\frac{4 \eta^{2}}{R_{0}^{2} \rho}\right\} .
\end{aligned}
$$

Hence, noting that $R_{\mathrm{d}}>0$, antibubbles must have higher resonance frequencies than regular bubbles.

\section{Methods}

We simulated the oscillating behaviour of gas bubbles and antibubbles in a viscous liquid. The shell was ignored, making it feasible to only compare the change in dynamics resulting from the addition of a droplet inside the bubble. An equilibrium bubble diameter of $5.0 \mu \mathrm{m}$ 
was chosen, which is representative for the current generation of ultrasound contrast agents used in clinical studies. The core droplet size within the antibubbles was varied between 0.4 (low) and 0.8 (high) times the bubble diameter. A single excitation pulse consisting of an amplitude-modulated sine-wave burst of 5 cycles at $2.5 \mathrm{MHz}$ was used. Mechanical indices (MI) of 0.1 (low) and 0.3 (high), equivalent to peak-to-peak acoustic pressures of 0.3 and $1 \mathrm{MPa}$, respectively, were evaluated. These acoustic pressures were chosen, because they match the acoustic conditions acceptable in clinical diagnostic imaging. Computations were performed using MATLAB 2013a (The Mathworks, Inc., Natick, MA, USA). The following fixed parameters were used: $p_{0}=1.0 \mathrm{~atm}, p_{\mathrm{v}}=2.3 \mathrm{kPa}, \gamma=1.4, \eta=1.0 \mathrm{mPa} \mathrm{s}$, $\rho=998 \mathrm{~kg} / \mathrm{m}^{3}$, and $\sigma=0.072 \mathrm{~N} / \mathrm{m}$. Frequency spectra of the radius-time curves were computed using the FFT algorithm in MATLAB.

\section{Results and discussion}

Figure 2 compares the resonance frequencies of antibubbles with load volumes of 40,60 , and $80 \%$, respectively. In all cases, as the diameter of the bubble decreased, the resonance frequency increased. An exponential-like increase in the resonance frequency can be seen as the core size increased. For a $2 \mu \mathrm{m}$ radius gas bubble the resonance frequency is $2.0 \mathrm{MHz}$. In the case of the 40,60 , and $80 \%$-loaded bubbles, the resonance frequencies would be $2.1,2.3$, and $3.0 \mathrm{MHz}$, respectively. All these frequencies are within the clinical diagnostic regime, thus indicating that these bubbles may have potential as an ultrasound contrast agent.

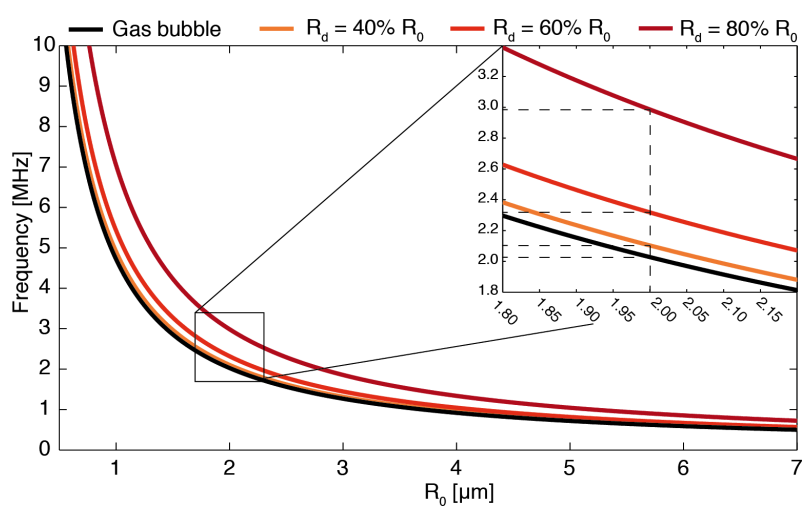

Fig. 2. Resonance frequencies of antibubbles with three different core sizes and free gas bubbles.

Figure 3 shows the simulated radius-time curves and equivalent frequency spectra, comparing antibubbles and gas bubbles. The upper panel compares the microbubble's maximum diameter during and after acoustic excitation. At a low MI and a low core volume (Fig. 3.1a), the oscillation behaviour is almost identical. During the second and third cycle of excitation, the antibubble has a larger excursion than the gas bubble. Both gas bubble
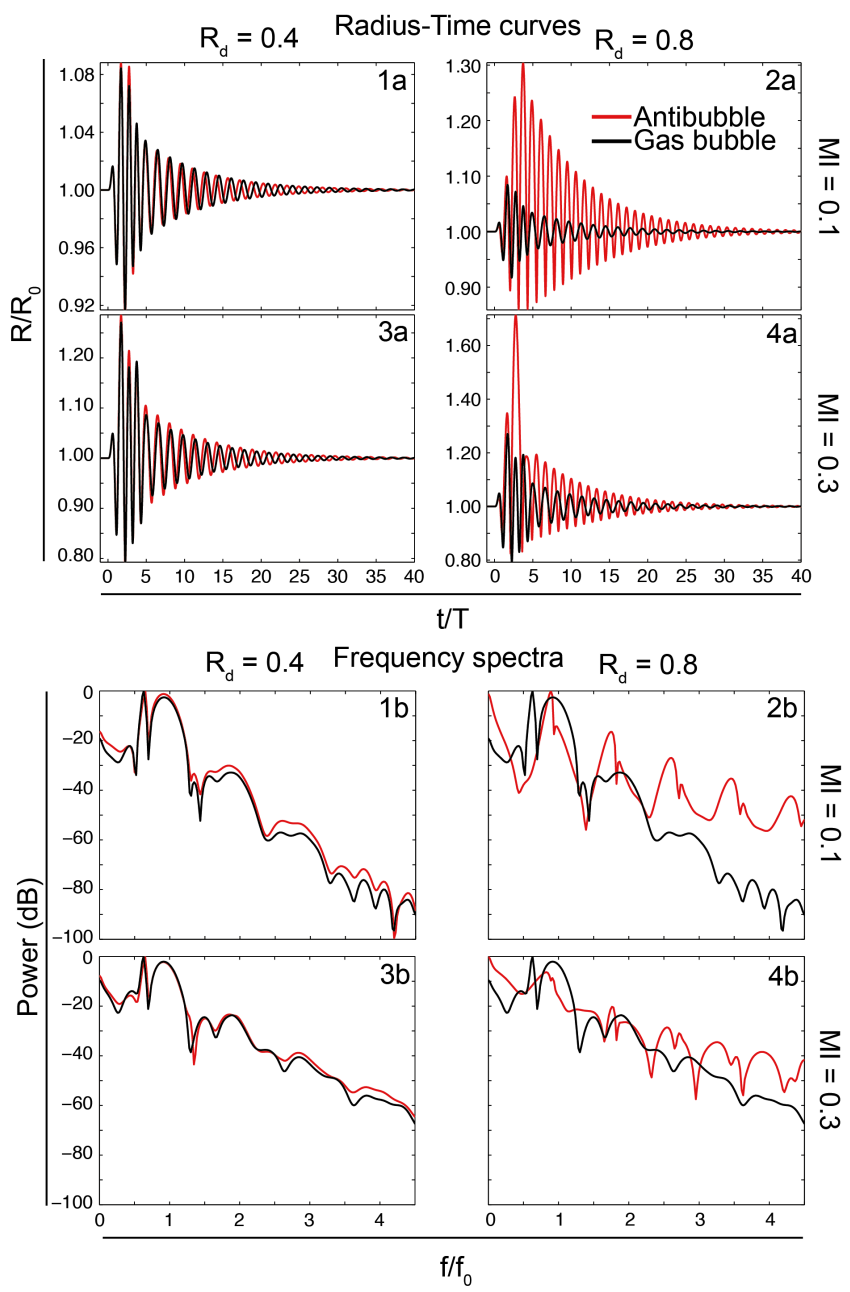

Fig. 3. Radius-time curves and their respective frequency spectra of antibubbles and gas bubbles.

and antibubble oscillate at the excitation frequency in phase. Once the excitation stops, i.e., by cycle 6 , a small phase shift can be seen. This phase shift continues to increase as the oscillations slowly dampen. The phase shift is evident by cycle 10 . This phase shift indicates that the antibubble has a higher resonance frequency than the gas bubble, as indicated by Fig. 2. When looking at the frequency spectrum of these oscillations (Fig. 3.1b), only a small increase in the higher harmonic components can be seen (between 2 and $8 \mathrm{~dB}$ ). Increasing the MI to 0.3 shows minimal change in behaviour (Fig. 3.3a). The initial excursion of the antibubble is once again larger than that of the gas bubble. However, this larger excursion is continuous for several more cycles. Nevertheless, the frequency spectrum (Fig. 3.3b) shows almost no difference between the antibubble and the gas bubble. The larger initial excursion may improve acoustic backscatter generated by these antibubbles when performing diagnostic ultrasound imaging.

Increasing the core volume to $80 \%$ provided a significant change in bubble behaviour. At a low MI with 
a high core load (Fig. 3.2a) the radial excursion of the antibubble was significantly larger than the gas bubble. This increased excursion was prominent until the oscillations completely decayed. In this case, the phase shift is also seen at an earlier time point, i.e., during excitation. This may be due to the inertia of the bubble being higher than the driving acoustic pressure. Due to the core size, the radius of the bubble never decreased below $80 \%$ of its original volume, resulting in highly asymmetric oscillations, indicating the positive excursion is much larger than the negative excursion.

The frequency spectrum (Fig. 3.2b) shows a noteworthy difference in both bandwidth and higher harmonic components when comparing the gas bubble to the antibubble. The inertial-driven oscillations of the antibubble resulted in narrow-bandwidth harmonic components with amplitudes substantially larger than the gas bubble. At the second harmonic, the antibubble had an $19 \mathrm{~dB}$ higher-frequency component, and at the third harmonic, the antibubble had a $30 \mathrm{~dB}$ higher-frequency component.

This increase in the higher-frequency content may be of use when attempting to distinguish loaded antibubbles from gas bubbles using a clinical diagnostic ultrasound scanner in nonlinear imaging modes [10].

Our simulations indicated that this increase of the acoustic pressure reduces the differences in oscillation amplitude and higher frequency components (Fig. 3.4a and $b)$.

Further simulations showed that a significant difference $(>6 \mathrm{~dB})$ in harmonic content was seen when the core size was $50 \%$ or greater than the antibubble diameter.

Overall, these results indicate that it is possible to simulate the radial response of a microbubble with an incompressible core, i.e., an antibubble. Our results also indicate that such microbubbles have larger oscillation amplitudes that increase with core size. Furthermore, as the core sizes increase, so do the higher-frequency components. In theory, this could be used to detect and distinguish antibubbles from microbubbles. Also, the increased oscillation amplitudes, and associated boundary speeds, of antibubbles may have an effect on the core. Such increased boundary speeds may vaporise liquid cores of the antibubbles, releasing it on demand, resulting in an ideal theranostic agent.

Consequently, studies on antibubble core release must be performed to confirm this hypothesis. Moreover, in our simulations the effect of a shell was neglected. Adding a shell would dampen the volumetric oscillations, potentially affecting the maximum bubble excursions and the harmonic components.

\section{Conclusions}

In our work, we show that antibubble dynamics are similar to gas bubble dynamics if the liquid core is less than $50 \%$ of the bubble diameter. However, if the core is more than $70 \%$ of the bubble diameter, at moderate and low acoustic pressures, the nonlinear behaviour dominates the oscillations. At higher acoustic pressures, differences in harmonic content are negligible.

\section{Acknowledgments}

This study has been supported by MedViz, an interdisciplinary research cluster from Bergen, Norway, by Haukeland University Hospital, University of Bergen, and Christian Michelsen Research AS.

\section{References}

[1] EFSUMB - European Course Book, Ed. C.F. Dietrich, EFSUMB, London 2012.

[2] M. Postema, O.H. Gilja, Curr. Pharm. Biotechnol. 8, 355 (2007).

[3] S. Kotopoulis, A. Delalande, M. Popa, V. Mamaeva, G. Dimcevski, O.H. Gilja, M. Postema, B.T. Gjertsen, E. Mc Cormack, Mol. Imag. Biol. 16, 52 (2014).

[4] S. Kotopoulis, G. Dimcevski, O.H. Gilja, D. Hoem, M. Postema, Med. Phys. 40, 072902 (2013).

[5] M. Postema, O.H. Gilja, World J. Gastroenterol. 17, 28 (2011).

[6] D.J. May, J.S. Allen, K.W. Ferrara, IEEE Trans. Ultrason. Ferroelectr. Freq. Control 49, 1400 (2002).

[7] M.J. Shortencarier, P.A. Dayton, S.H. Bloch, P.A. Schumann, T.O. Matsunaga, F.W. Ferrara, IEEE Trans. Ultrason. Ferroelectr. Freq. Control 51, 822 (2004).

[8] M. Postema, F.J. ten Cate, G. Schmitz, N. de Jong, A. van Wamel, Lett. Drug Des. Discov. 4, 74 (2007).

[9] A.T. Poortinga, Langmuir 27, 2138 (2011).

[10] J.E. Silpe, J.K. Nunes, A.T. Poortinga, H.A. Stone, Langmuir 29, 8782 (2013).

[11] M. Postema, Fundamentals of Medical Ultrasonics, Spon Press, New York 2011. 